\title{
Evidence for Hierarchical Black Hole Mergers in the Second LIGO-Virgo Gravitational Wave Catalog
}

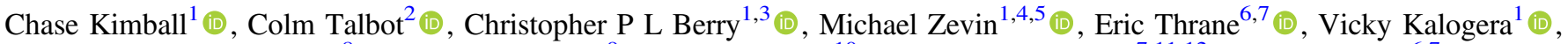

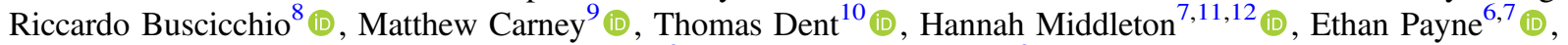 \\ John Veitch ${ }^{3}$ (iD), and Daniel Williams ${ }^{3}$ (D) \\ ${ }^{1}$ Center for Interdisciplinary Exploration and Research in Astrophysics (CIERA), Department of Physics and Astronomy, Northwestern University, 1800 Sherman \\ Avenue, Evanston, IL 60201, USA; CharlesKimball2022@u.northwestern.edu \\ ${ }^{2}$ LIGO Laboratory, California Institute of Technology, Pasadena, CA 91125, USA \\ ${ }^{3}$ SUPA, School of Physics and Astronomy, University of Glasgow, Glasgow G12 8QQ, UK \\ ${ }^{4}$ Kavli Institute for Cosmological Physics, The University of Chicago, 5640 South Ellis Avenue, Chicago, IL 60637, USA \\ ${ }^{5}$ Enrico Fermi Institute, The University of Chicago, 933 East 56th Street, Chicago, IL 60637, USA \\ ${ }^{6}$ School of Physics and Astronomy, Monash University, VIC 3800, Australia \\ ${ }^{7}$ OzGrav: The ARC Centre of Excellence for Gravitational-Wave Discovery, Clayton, VIC 3800, Australia \\ ${ }^{8}$ Institute for Gravitational Wave Astronomy and School of Physics and Astronomy, University of Birmingham, Birmingham B15 2TT, UK \\ ${ }_{9}$ Department of Physics, Washington University, One Brookings Drive, St. Louis, MO 63130, USA \\ ${ }^{10}$ Instituto Galego de Física de Altas Enerxías, Universidade de Santiago de Compostela, E-15782, Spain \\ ${ }^{11}$ Centre for Astrophysics and Supercomputing, Swinburne University of Technology, Hawthorn, VIC 3122, Australia \\ ${ }^{12}$ School of Physics, University of Melbourne, Parkville, VIC 3010, Australia \\ Received 2021 May 7; revised 2021 June 9; accepted 2021 June 11; published 2021 July 14
}

\begin{abstract}
We study the population properties of merging binary black holes in the second LIGO-Virgo Gravitational-Wave Transient Catalog assuming they were all formed dynamically in gravitationally bound clusters. Using a phenomenological population model, we infer the mass and spin distribution of first-generation black holes, while self-consistently accounting for hierarchical mergers. Considering a range of cluster masses, we see compelling evidence for hierarchical mergers in clusters with escape velocities $\gtrsim 100 \mathrm{~km} \mathrm{~s}^{-1}$. For our most probable cluster mass, we find that the catalog contains at least one second-generation merger with $99 \%$ credibility. We find that the hierarchical model is preferred over an alternative model with no hierarchical mergers (Bayes factor $\mathcal{B}>1400$ ) and that GW190521 is favored to contain two second-generation black holes with odds $\mathcal{O}>700$, and GW190519, GW190602, GW190620, and GW190706 are mixed-generation binaries with $\mathcal{O}>10$. However, our results depend strongly on the cluster escape velocity, with more modest evidence for hierarchical mergers when the escape velocity is $\lesssim 100 \mathrm{~km} \mathrm{~s}^{-1}$. Assuming that all binary black holes are formed dynamically in globular clusters with escape velocities on the order of tens of $\mathrm{km} \mathrm{s}^{-1}$, GW190519 and GW190521 are favored to include a second-generation black hole with odds $\mathcal{O}>1$. In this case, we find that $99 \%$ of black holes from the inferred total population have masses that are less than $49 M_{\odot}$, and that this constraint is robust to our choice of prior on the maximum black hole mass.
\end{abstract}

Unified Astronomy Thesaurus concepts: Gravitational wave sources (677); Gravitational wave astronomy (675); Astrophysical black holes (98); Hierarchical models (1925)

\section{Introduction}

The second LIGO-Virgo Gravitational Wave Transient Catalog (GWTC-2) has significantly expanded our set of gravitational wave (GW) observations (Abbott et al. 2021a). It contains a total of 46 binary black hole (BBH) candidates, excluding GW190814 (Abbott et al. 2020a) whose source could also be a neutron starblack hole binary, whereas the previous catalog only contained 10 BBHs (Abbott et al. 2019). Multiple astrophysical formation channels have been suggested to explain the population of BBHs, and each of these have uncertainties in their underlying physics (e.g., Kruckow et al. 2016; Rodriguez et al. 2016; Abbott et al. 2016a; Klencki et al. 2018; Sasaki et al. 2018; Kumamoto et al. 2020; Tagawa et al. 2020; Tang et al. 2020; Di Carlo et al. 2020b; Zevin et al. 2020b). GW observations can constrain the relative contribution of formation channels and their uncertain physics, and as the catalog grows these constraints become more precise (Stevenson et al. 2017; Talbot \& Thrane 2017; Vitale et al. 2017; Zevin et al. 2017; Barrett et al. 2018; Fishbach et al. 2018; Zevin et al. 2021).

Among the GWTC-2 systems there are high-mass BBHs that have components with masses of $\gtrsim 45 M_{\odot}$ (Abbott et al. 2021a), the most massive being the source of GW190521 (Abbott et al. 2020b). Black holes of $\sim 45-135 M_{\odot}$ are not typically expected to form via standard stellar evolution as the pair-instability process either limits the maximum mass of the progenitor star's core or completely disrupts the star entirely (Fryer et al. 2001; Heger \& Woosley 2002; Belczynski et al. 2016; Spera \& Mapelli 2017; Farmer et al. 2019; Stevenson et al. 2019; Farmer et al. 2020; Woosley \& Heger 2021). Potential (non-mutually exclusive) astrophysical formation mechanisms for black holes in this mass gap include hierarchical mergers, where the remnant of a previous merger becomes part of a new binary (Miller \& Hamilton 2002; Antonini \& Rasio 2016; Gerosa \& Berti 2017; Rodriguez et al. 2019; Yang et al. 2019; Anagnostou et al. 2020; Fragione \& Silk 2020; Mapelli et al. 2020; Fragione et al. 2020a; Banerjee 2021); stellar mergers, which may result in a larger hydrogen envelope around a core below the pairinstability threshold (Spera et al. 2018; Kremer et al. 2020; Di Carlo et al. 2020a; González et al. 2021); formation of black holes from Population III stars that are able to retain their hydrogen envelopes (Farrell et al. 2021; Kinugawa et al. 2021; 

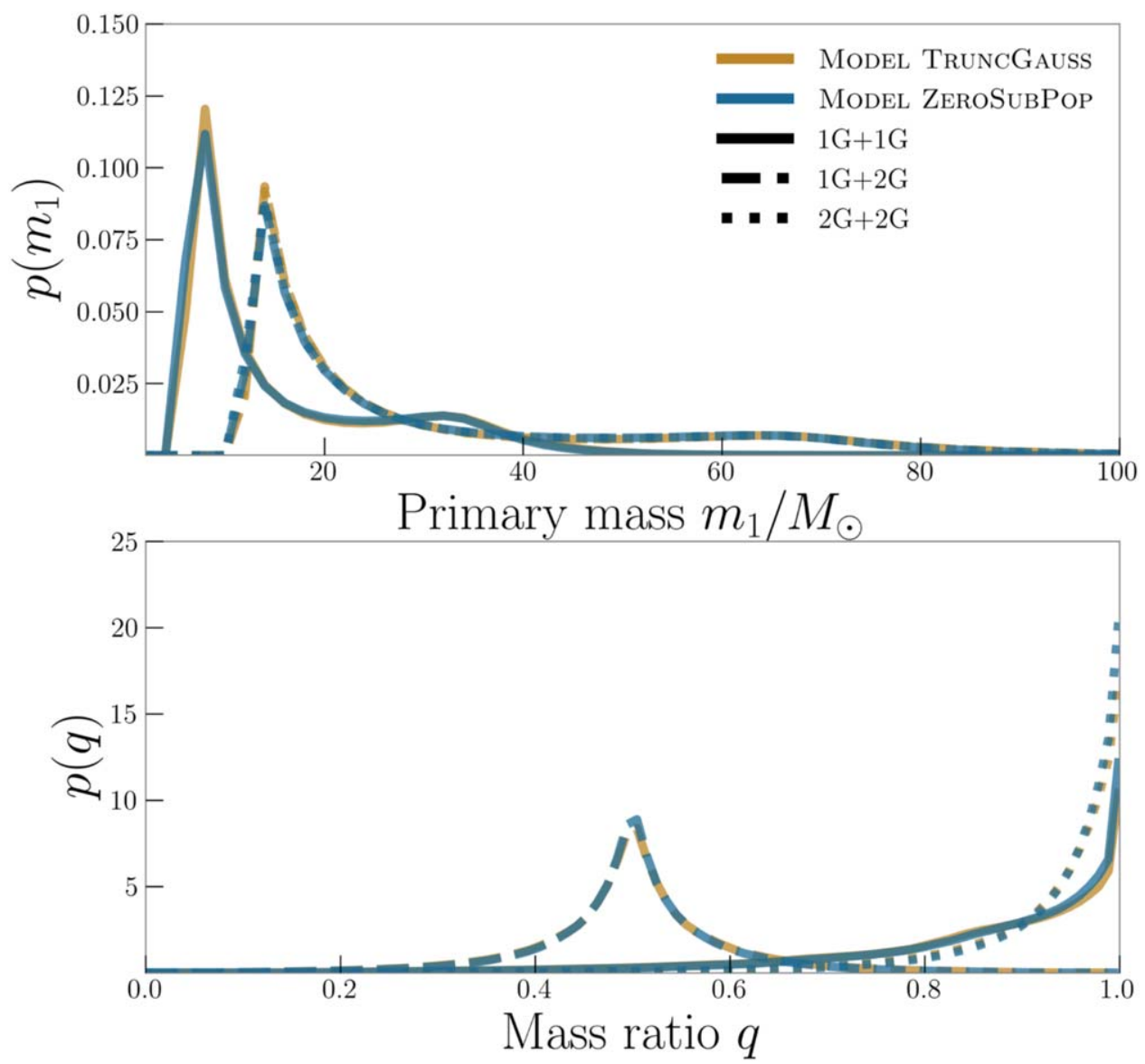

Figure 1. Posterior predictive distributions for the primary mass $m_{1}$ and mass ratio $q$. The solid, dashed, and dotted lines indicate the $1 \mathrm{G}+1 \mathrm{G}, 1 \mathrm{G}+2 \mathrm{G}$, and $2 \mathrm{G}+2 \mathrm{G}$ distribution, respectively. In blue, we plot the distributions inferred when modeling the $1 \mathrm{G}$ spin distribution as a non-singular Beta distribution together with a delta function at zero. In orange, we plot the distributions inferred when using a truncated Gaussian.

Vink et al. 2021), formation via stellar triples in the field (Vigna-Gómez et al. 2021); growth via accretion in an active galactic nucleus (AGN) disk (McKernan et al. 2012; Michaely \& Perets 2020; Secunda et al. 2020; Tagawa et al. 2020 ), or growth via rapid gas accretion in dense primordial clusters (Roupas \& Kazanas 2019).

Hierarchical mergers in globular clusters were considered as an origin for GW190521 (Abbott et al. 2020c) with inconclusive results. However, hints of eccentricity in follow-up analyses of GW190521 add weight to this explanation (Gayathri et al. 2020; Romero-Shaw et al. 2020a). To confidently identify hierarchical mergers, it is important to study events in the context of a population model that fits the mass distribution (and any mass cutoffs) for the first-generation $(1 \mathrm{G})$ black holes not formed through mergers (Doctor et al. 2020; Sedda et al. 2020; Kimball et al. 2020a; Tiwari \& Fairhurst 2021).

We apply the population inference framework from Kimball et al. (2020b) to analyze the BBHs from GWTC-2. This framework assumes a phenomenological population model based on simulations of metal-poor globular clusters from Rodriguez et al. (2019). Considering a fiducial set of globular cluster masses, we simultaneously infer the properties of the $1 \mathrm{G}+1 \mathrm{GBBH}$ population-whose remnants are secondgeneration $(2 \mathrm{G})$ black holes - and the relative merger rates of hierarchical mergers. The expanded catalog enables the population parameters, including the mass distribution, to be more precisely determined (Abbott et al. 2021b). We find that several of the BBHs are likely to be the results of hierarchical mergers: the leading candidates are GW190519_153544 (GW190519) and GW190521.

In Section 2 we review the key components of our population inference framework; the results of this are given in Section 3, with additional description of the population hyperparameters in the Appendix, and we discuss our findings in Section 4.

\section{Methods}

We perform Bayesian hierarchical inference to infer the the population properties of BBHs following Kimball et al. (2020b). We employ phenomenological models for the mass and spin distributions of $1 \mathrm{G}+1 \mathrm{G}, 1 \mathrm{G}+2 \mathrm{G}$, and $2 \mathrm{G}+2 \mathrm{G}$ BBHs merging in a dense stellar environment; see Figures 1 and 2. The $1 \mathrm{G}+1 \mathrm{G}$ model is nearly identical to population models used in Abbott et al. (2021b): it is equivalent to the POWER LAW + PEAK mass model (but omits the low-mass smoothing and adopts a Gaussian prior on the maximum mass cut-off) and is similar to the DEFAULT spin model. We consider two separate modifications to that spin model: one that adds a parameter that allows for a subpopulation of zero-spin BBHs, and one consisting of a truncated Gaussian with a broad prior on the mean that allows for distributions with sharp peaks at 0 or 1; we refer to these as MODEL ZEROSUBPOP and MODEL TRUNCGAUSS, respectively. The particulars of the mass and spin models are discussed further in the Appendix. 

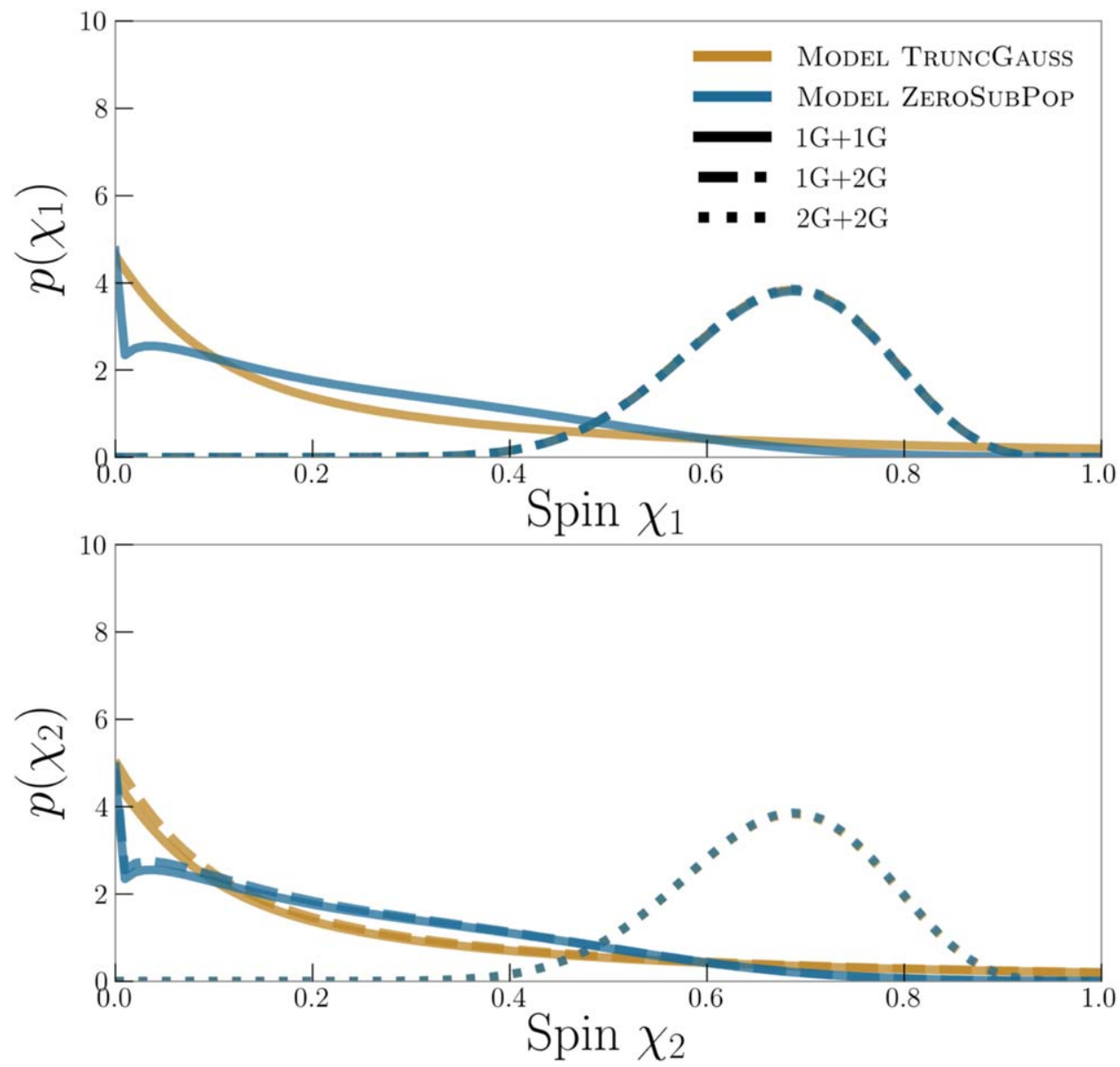

Figure 2. Posterior predictive distributions for the component black hole spins. The solid, dashed, and dotted lines indicate the $1 \mathrm{G}+1 \mathrm{G}, 1 \mathrm{G}+2 \mathrm{G}$, and $2 \mathrm{G}+2 \mathrm{G}$ distribution, respectively. In blue, we plot the distributions inferred when modeling the $1 \mathrm{G}$ spin distribution as a non-singular Beta distribution together with a delta function at zero. In orange, we plot the distributions inferred when using a truncated Gaussian.

The $2 \mathrm{G}$ black holes are assumed to be roughly twice the mass of $1 \mathrm{G}$ black holes; the mass ratio distribution for $1 \mathrm{G}+2 \mathrm{G}$ binaries is peaked around $q \sim 1 / 2$ while the $2 \mathrm{G}+2 \mathrm{G}$ distribution is similar to the $1 \mathrm{G}+1 \mathrm{G}$ model but with an increased preference for near equal-mass binaries to account for the more massive components in a strong encounter forming bound binaries (Sigurdsson \& Phinney 1993; Heggie et al. 1996; Downing et al. 2011). The 1G $+2 \mathrm{G}$ and $2 \mathrm{G}+2 \mathrm{G}$ spin models presume that $2 \mathrm{G}$ black holes have dimensionless spin $\chi \approx 0.67$ inherited from the orbital angular momentum of the progenitor binary (Pretorius 2005; Gonzalez et al. 2007; Buonanno et al. 2008). The population models are described as conditional priors $\pi(\theta \mid \Lambda)$ where $\theta$ are the parameters of a single binary (e.g., mass and spin) while $\Lambda$ refers to the population hyperparameters describing the shape of the mass and spin distributions (e.g., the power-law index of the primary black hole mass spectrum). Our goal is two-fold: estimate the population hyperparameters $\Lambda$ and carry out model selection to evaluate the Bayesian odds that events in GWTC-2 are formed hierarchically.

The relative rates of $1 \mathrm{G}+1 \mathrm{G}, 1 \mathrm{G}+2 \mathrm{G}$, and $2 \mathrm{G}+2 \mathrm{G}$ mergers depend upon the properties of their cluster environment as well as the masses and spins of the BBH population. GW recoil kicks may lead to remnants being ejected from a cluster; kick magnitudes are strongly dependent on progenitor spins with larger spins leading to larger kicks (Campanelli et al. 2007; Gonzalez et al. 2007; Bruegmann et al. 2008; Lousto \& Zlochower 2011; Varma et al. 2019), as well as the mass ratio of the merging binary. We calculate the fraction of retained merger remnants $F_{\text {ret }}$ given the population properties of the $1 \mathrm{G}$ black holes and assuming a cluster described by a Plummer potential (Plummer 1911) mass of $M_{\mathrm{c}}$ with Plummer radius $r_{\mathrm{c}}$. For our default cluster, we assume that $M_{\mathrm{c}}=5 \times 10^{5} M_{\odot}$ and $r_{\mathrm{c}}=1 \mathrm{pc}$, corresponding to a central escape velocity of $\sim 65 \mathrm{~km} \mathrm{~s}^{-1}$. We assume that the relative merger rates scale as $R_{1 \mathrm{G}+2 \mathrm{G}} / R_{1 \mathrm{G}+1 \mathrm{G}} \propto F_{\text {ret }}, R_{2 \mathrm{G}+2 \mathrm{G}} / R_{\mathrm{IG}+1 \mathrm{G}} \propto F_{\text {ret }}^{2}$, with the constant of proportionality calibrated against globular cluster simulations (Rodriguez et al. 2019).

For our analysis, we consider the $44 \mathrm{BBH}$ (excluding GW190814, for which the nature of the secondary component is unknown) used in the GWTC-2 population analysis (Abbott et al. 2021b). For GWTC-1 events, we use the same single-event posterior samples as Kimball et al. (2020b), for GW190412 we use samples from Zevin et al. (2020a), for GW190521 we use the preferred samples from Abbott et al. (2020c), and for the other GWTC-2 events we use the public samples from Abbott et al. (2021a). ${ }^{13}$ For the new GWTC-2 events we use results calculated with the IMRPHENOMD and IMRPHENOMPV2 waveforms (Hannam et al. 2014; Khan et al. 2016). We generate posterior samples for population hyperparameters $\Lambda$ using the nested sampler DYNESTY (Speagle 2020) using the GWPOPULATION framework (Talbot et al. 2019), which takes

\footnotetext{
13 Posterior samples for GW190412, GW190521, and the other GWTC-2 systems are available from https://doi.org/10.5281/zenodo.3900546, https://doi.org/10. 7935/1502-wj52 and https://doi.org/10.7935/99gf-ax93, respectively.
} 


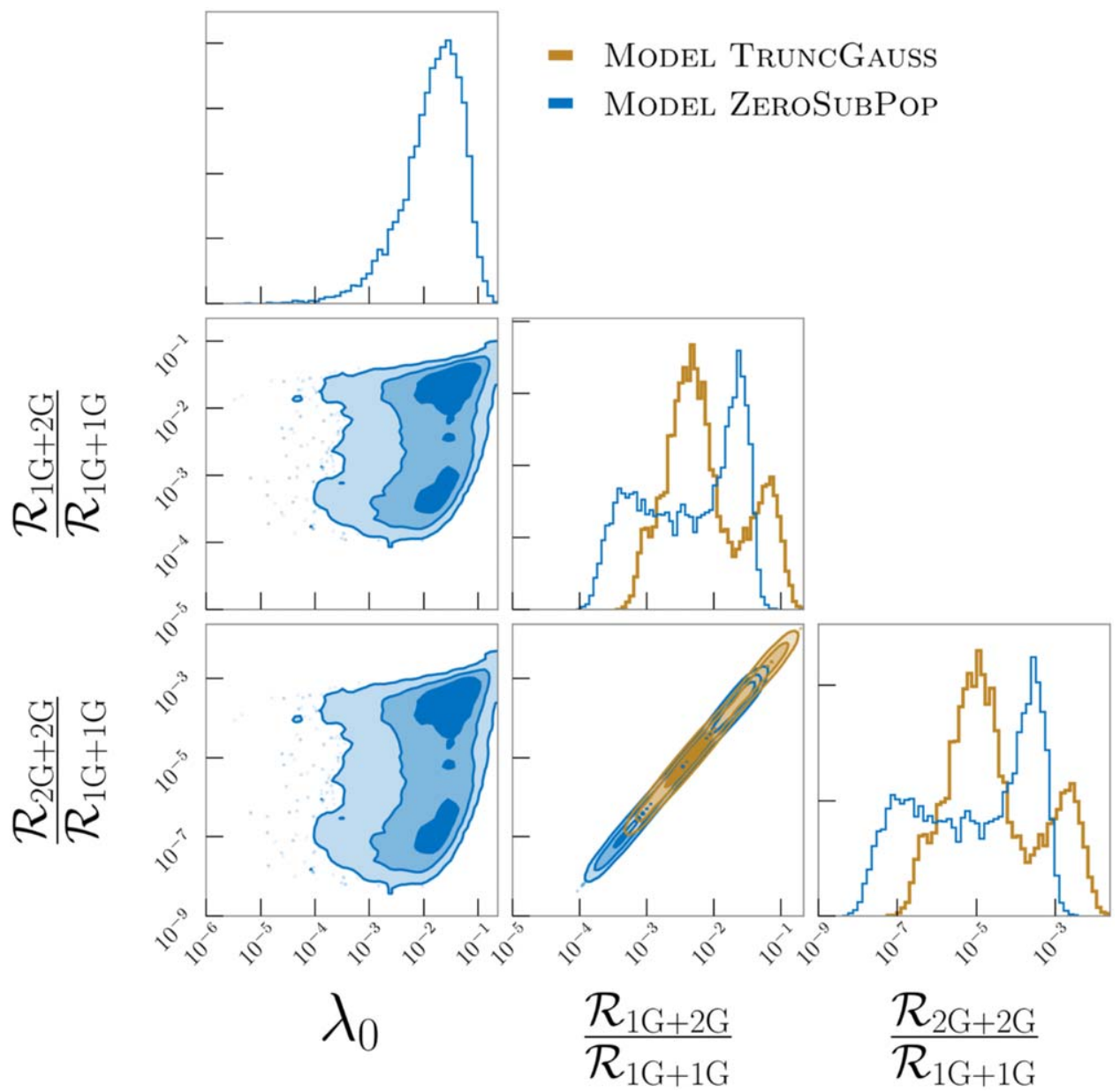

Figure 3. Posteriors of the inferred branching ratios, and the fraction $\lambda_{0}$ of $1 \mathrm{G}+1 \mathrm{G}$ black holes with zero spin for MODEL ZEROSUBPOP. The branching ratios give the relative $1 \mathrm{G}+2 \mathrm{G}$ vs. $1 \mathrm{G}+1 \mathrm{G}$ and $2 \mathrm{G}+2 \mathrm{G}$ vs. $1 \mathrm{G}+1 \mathrm{G}$ merger rates. We plot the results using MODEL TRUNCGAUSS and MODEL ZEROSUBPOP in orange and blue, respectively.

advantage of BILBY (Ashton et al. 2019; Romero-Shaw et al. 2020b).

\section{Application to GWTC-2}

\subsection{Inferred Populations}

Applying our analysis to the 44 BBH candidates in GWTC-2 analyzed in Abbott et al. (2021b), we infer population hyperparameters for our mass and spin models (Figures 6, Figure 7, and Figure 8 in the Appendix). For both MODEL TRUNCGAUSS and MODEL ZEROSUBPOP, we find that including the $1 \mathrm{G}+2 \mathrm{G}$ and $2 \mathrm{G}+2 \mathrm{G}$ population components is preferred, finding Bayes factors of 5 and 7, respectively, in favor of including versus excluding the hierarchical components.

In our inferred $1 \mathrm{G}$ mass distribution, the mean of the Gaussian mass component is well constrained to $\mu_{m}=32.0_{-6.8}^{+8.5} M_{\odot}$ and $\mu_{m}=31.5_{-9.0}^{+23.0} M_{\odot}$ for MODEL TRUNCGAUSS and MODEL ZEROSUBPOP, respectively. For both models we recover our prior on the maximum mass cut-off $m_{\max }$. In Figures 1 and 2, we plot the posterior predictive distributions for the $1 \mathrm{G}+1 \mathrm{G}, 1 \mathrm{G}+2 \mathrm{G}$, and $2 \mathrm{G}+2 \mathrm{G}$ populations. Using MODEL TRUNCGAUSS (MODEL ZEROSUBPOP), we find that $99 \%$ of $1 \mathrm{G}+1 \mathrm{G}$ black holes are less than $47 M_{\odot}\left(47 M_{\odot}\right)$, and $99 \%$ of black holes in the total population are less than $49 M_{\odot}\left(48 M_{\odot}\right)$, consistent with the results of Kimball et al. (2020b). These upper limits are lower than those found for the POWER LAW + PEAK model in Abbott et al. (2021b), but that model does not include a high-mass hierarchical component, and requires a flatter power law to fit the heavier black holes in GWTC-2. Relaxing the prior on the maximum mass cut-off to a uniform prior out to $100 M_{\odot}$ (Figures 9, 10, and 11), we do not obtain stringent constraints on $m_{\max }$, but find that it peaks around $\sim 80 M_{\odot}$. In this case, we find that $99 \%$ of $1 \mathrm{G}+1 \mathrm{G}$ black holes are less than $49 M_{\odot}\left(49 M_{\odot}\right)$, and $99 \%$ of black holes in the total population are less than $51 M_{\odot}\left(50 M_{\odot}\right)$.

Using MODEL TRUNCGAUSS and MODEL ZEROSUBPOP, $90 \%$ of $1 \mathrm{G}+1 \mathrm{G}$ black holes have spins less than 0.65 and 0.50 , respectively. With MODEL ZEROSUBPOP, the fraction $\lambda_{0}$ of BBHs originating from the zero-spin channel is constrained to be less than 0.12 at the $99 \%$ credible level.

\subsection{Relative Merger Rates}

GW recoil kick velocities generally increase with the spin magnitudes of merging black holes. Figure 2 illustrates that while the spin distribution inferred using MODEL TRUNCGAUSS does not explicitly include a subpopulation at $\chi=0$, a larger portion of the population has spins less than $\sim 0.1$ than for MODEL ZEROSUBPOP, which results in lower typical recoil velocities and hence higher relative hierarchical merger rates. In Figure 3, we plot the posteriors for these rates, as well as for the fraction $\lambda_{0}$ of $1 \mathrm{G}+1 \mathrm{G}$ black holes in MODEL ZEROSUBPOP with zero spin. 


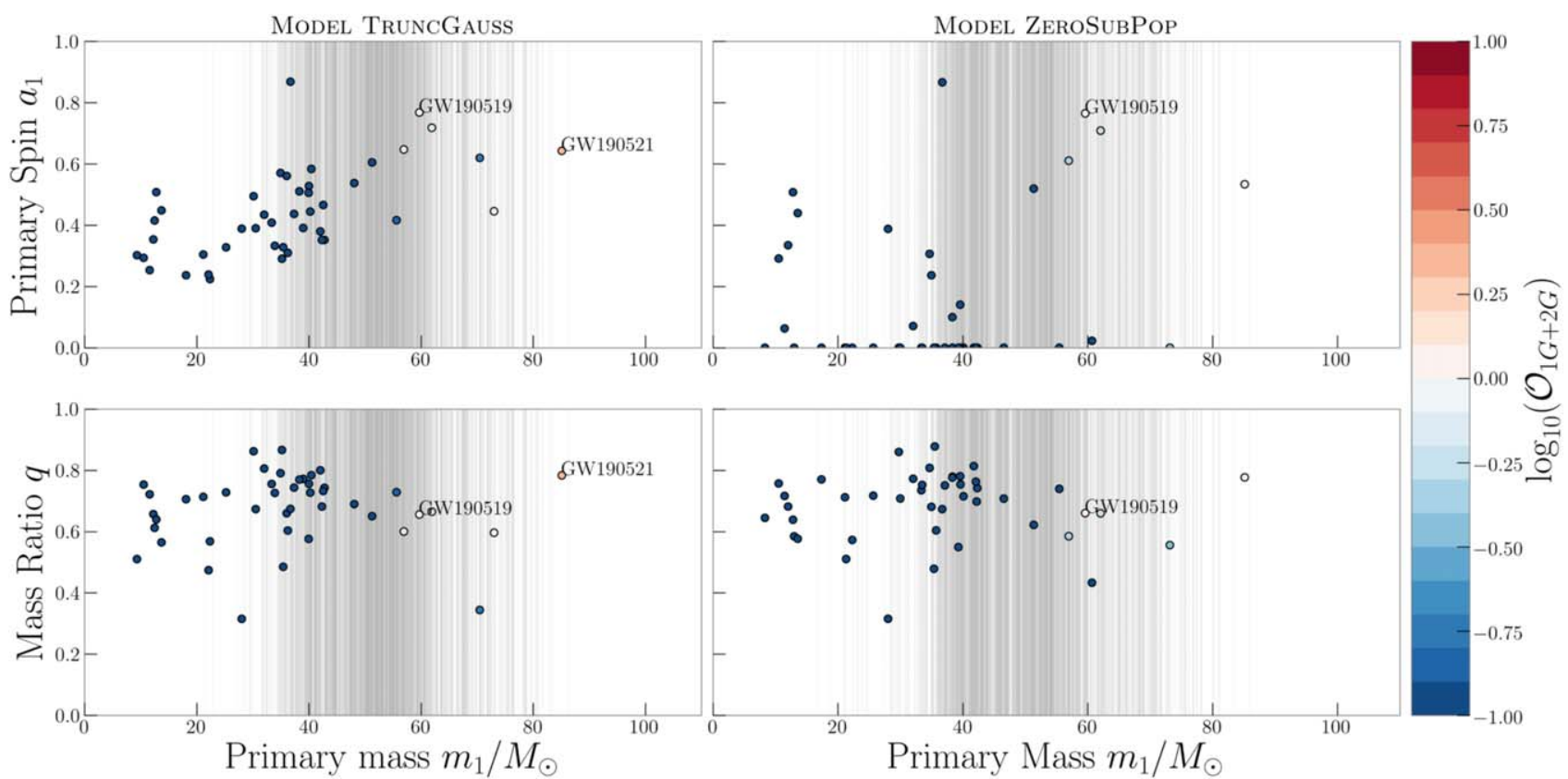

Figure 4. Odds of events in GWTC-2 having $1 \mathrm{G}+2 \mathrm{G}$ vs. $1 \mathrm{G}+1 \mathrm{G}$ origin, as a function of the inferred median primary black hole mass, mass ratio, and primary black hole spin. The results using MODEL TRUNCGAUSS and MODEL ZEROSUBPOP are plotted on the left and right, respectively. The gray vertical lines are draws from the corresponding inferred posterior over the maximum $1 \mathrm{G}$ black hole mass.

Using MODEL TRUNCGAUSS, we infer median relative rates $\mathcal{R}_{1 \mathrm{G}+2 \mathrm{G}} / \mathcal{R}_{1 \mathrm{G}+1 \mathrm{G}}=5.8 \times 10^{-3}$ and $\mathcal{R}_{2 \mathrm{G}+2 \mathrm{G}} / \mathcal{R}_{1 \mathrm{G}+1 \mathrm{G}}=$ $1.7 \times 10^{-5}$ with $99 \%$ upper limits of 0.12 and $7.6 \times 10^{-3}$, respectively. Taking into account selection effects, the detected population would have median relative rates of $\mathcal{R}_{1 \mathrm{G}+2 \mathrm{G}}^{\mathrm{det}} /$ $\mathcal{R}_{1 \mathrm{G}+1 \mathrm{G}}^{\mathrm{det}}=0.01$ and $\mathcal{R}_{2 \mathrm{G}+2 \mathrm{G}} / \mathcal{R}_{1 \mathrm{G}+1 \mathrm{G}}=7.0 \times 10^{-5}$ with $99 \%$ upper limits of 0.23 and 0.03 , respectively. Using MODEL ZEROSUBPOP, these rates become $5.3 \times 10^{-3}(0.01)$ and $1.4 \times 10^{-5}\left(5.7 \times 10^{-5}\right)$, with $99 \%$ upper limits of $0.04(0.08)$ and $9.8 \times 10^{-4}\left(4.0 \times 10^{-3}\right)$, respectively, for the astrophysical (detected) population. The median inferred relative rates are roughly twice those found using the same model in Kimball et al. (2020b), though consistent with the upper limits reported there. The results for both models are consistent with the results of Monte Carlo modeling of black hole populations in globular clusters: Rodriguez et al. (2019) found that the $\approx 14 \%$ of merging BBHs from the underling population in their models contain $2 \mathrm{G}$ black holes in the extreme case where all $1 \mathrm{G}$ black holes have zero spin (this fraction drops to $\lesssim 1 \%$ when they increase $1 \mathrm{G}$ black hole spins to $\chi=0.5$ ).

\subsection{Posterior Odds for Hierarchical Origin}

For each event in GWTC-2, we calculate the posterior odds $\mathcal{O}$ in favor of hierarchical versus $1 \mathrm{G}+1 \mathrm{G}$ origin. We plot the odds in favor of $2 \mathrm{G}+2 \mathrm{G}$ versus $1 \mathrm{G}+1 \mathrm{G}$ origin assuming MODEL TRUNCGAUSS and MODEL ZEROSUBPOP in Figure 4.

Assuming MODEL TRUNCGAUSS, we find that across all 44 BBHs in GWTC-2 the probability that at least one binary contains a 2G black hole is 99\%. GW190519 and GW190521 are most likely of $1 \mathrm{G}+2 \mathrm{G}$ origin, favored over a $1 \mathrm{G}+1 \mathrm{G}$ origin with $1.2: 1$ and $2: 1$ odds, respectively. We also favor a $2 \mathrm{G}+2 \mathrm{G}$ origin over $1 \mathrm{G}+1 \mathrm{G}$ for GW190521 with odds of 1.2:1. We find roughly even odds for GW190602_175927 (GW190602) and GW190706_ 222641 (GW190706) being of $1 \mathrm{G}+2 \mathrm{G}$ origin. As in Kimball et al. (2020b), we find that GW170729 is most likely of
$1 \mathrm{G}+1 \mathrm{G}$ origin, though at slightly higher odds of $1: 10$ of being of $1 \mathrm{G}+2 \mathrm{G}$ origin rather than $1 \mathrm{G}+1 \mathrm{G}$.

Using MODEL ZEROSUBPOP, which finds lower relative hierarchical merger rates, odds decrease across all events. We find that the probability that at least one binary in GWTC-2 contains a 2G black hole is $96 \%$. GW190519 is marginally favored to have a $1 \mathrm{G}+2 \mathrm{G}$ origin with $1.1: 1$ odds over a $1 \mathrm{G}$ $+1 \mathrm{G}$ origin. Meanwhile, GW190521 has roughly even odds of being $1 \mathrm{G}+2 \mathrm{G}$ versus $1 \mathrm{G}+1 \mathrm{G}$ at $1.0: 1$ odds, and a $2 \mathrm{G}$ $+2 \mathrm{G}$ origin is disfavored to a $1 \mathrm{G}+1 \mathrm{G}$ origin at $1: 4$ odds.

\subsection{Varying Cluster Parameters}

Our default Plummer model is chosen as representative of a typical globular cluster environment such as those in the vicinity of the Milky Way today, where central escape velocities are on the order of tens of kilometers per second (Baumgardt \& Hilker 2018). However, globular clusters in the Milky Way may have been up to a few times more massive at formation than at present (Webb \& Leigh 2015). Furthermore, hierarchical mergers may occur in a wide range of dynamical environments with significantly different escape velocities, including AGN disks and nuclear star clusters. Although our phenomenological models are tuned to the results of simulations of typical present-day globular clusters (Rodriguez et al. 2019), we can get an illustrative idea of how results scale with the mass and compactness of the assumed dynamical environment by varying the parameters of our simple Plummer model. We do not expect all BBHs to come from a single type of cluster, but our results let us explore a range of different average cluster sizes.

In Figure 5, we show results when considering models with Plummer masses $10^{4}-10^{9} M_{\odot}$ and radii $0.01-1 \mathrm{pc}$; both these parameters vary cluster escape velocities and thus the retention rate of hierarchical mergers. At low escape velocities $\left(\sim 10-50 \mathrm{~km} \mathrm{~s}^{-1}\right)$, almost no $1 \mathrm{G}+1 \mathrm{G}$ merger products are retained and the relative $1 \mathrm{G}+2 \mathrm{G}$ and $2 \mathrm{G}+2 \mathrm{G}$ rates are negligible. In this case, the inferred 

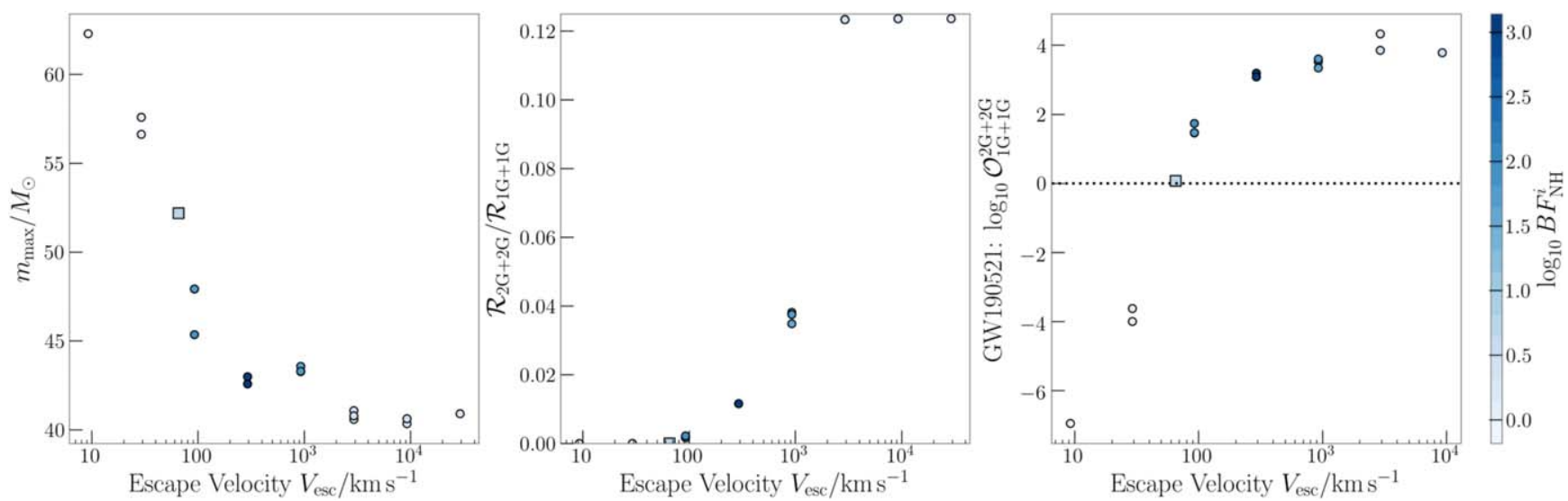

Figure 5. Inferred population properties as a function of central escape velocity using MODEL TRUNCGAUSS when considering models with Plummer masses $10^{4}$ $10^{9} M_{\odot}$ and radii $0.01-1 \mathrm{pc}$. In the left and middle panels, we plot the median inferred maximum black hole mass and relative $2 \mathrm{G}+2 \mathrm{G}$ vs. $1 \mathrm{G}+1 \mathrm{G}$ merger rate. In the right panel, we plot the odds in favor of GW190521 being a $2 \mathrm{G}+2 \mathrm{G}$ vs. $1 \mathrm{G}+1 \mathrm{G}$ merger. The points are shaded according to the Bayes factors in favor of the hierarchical model vs. a model excluding hierarchical channels $\mathrm{BF}_{\mathrm{NH}}$. The square marker indicates our default cluster model.

posterior on the maximum black hole mass $m_{\max }$ shifts away from the astrophysical prior toward higher masses in order to accommodate massive GWTC-2 events as $1 \mathrm{G}+1 \mathrm{GBBHs}$. As we move toward models with higher central escape velocities, the fraction of retained $1 \mathrm{G}+1 \mathrm{G}$ merger products and the relative $1 G+2 G$ and $2 G+2 G$ rates rapidly increase, and the odds in favor of GWTC-2 events being of hierarchical origin grow. It is expected that the rate of hierarchical mergers strongly depends on the escape velocity of their dynamical environments (Holley-Bockelmann et al. 2008; Moody \& Sigurdsson 2009; Antonini et al. 2019; Gerosa \& Berti 2019; Rodriguez et al. 2020; Sedda et al. 2020; Fragione et al. 2020b; Mapelli et al. 2021). We find that even a modest increase in the central escape velocity to $\sim 90 \mathrm{~km} \mathrm{~s}^{-1}$ leads to the conclusion that GW190521 is favored to be a $2 \mathrm{G}+2 \mathrm{G}$ versus $1 \mathrm{G}+1 \mathrm{G}$ merger at $>10: 1$ odds, and that the probability that at least one of the BBHs in GWTC-2 contains a $2 \mathrm{G}$ black hole is $>99.99 \%$.

We find that for all of our assumed cluster models, the events in GWTC-2 are better fit including the hierarchical channels than when excluding those channels (equivalent to setting $V_{\text {esc }}=0 \mathrm{~km} \mathrm{~s}^{-1}$ ), with the highest Bayes factors corresponding to models where the central escape velocities are $\sim 300 \mathrm{~km} \mathrm{~s}^{-1}$. In Figure 5, we show Bayes factors in favor of our hierarchical model versus a model with only $1 \mathrm{G}+1 \mathrm{G} B \mathrm{BH}$; taking the ratio of these Bayes factors gives cluster-wise Bayes factors comparing how well the data are supported by different cluster models. For clusters with escape velocities of $\sim 300 \mathrm{~km} \mathrm{~s}^{-1}$, which have the highest Bayes factors, we find that $99 \%$ of $1 \mathrm{G}+1 \mathrm{G}$ black holes are below $40 M_{\odot}\left(40 M_{\odot}\right)$ and that $99 \%$ of all black holes are below $67 M_{\odot}\left(66 M_{\odot}\right)$ using MODEL TRUNCGAUSS (MODEL ZEROSUBPOP). We infer median relative rates for MODEL TRUNCGAUSS (MODEL ZEROSUBPOP) of $1 \mathrm{G}+2 \mathrm{G}$ and $2 \mathrm{G}+2 \mathrm{G}$ versus $1 \mathrm{G}+1 \mathrm{G}$ mergers of 0.15 $(0.14)$ and $0.01\left(9.2 \times 10^{-3}\right)$ respectively, with $99 \%$ upper limits of $0.29(0.25)$ and $0.04(0.03)$. When accounting for selection effects, we infer median relative rates for the detected population with MODEL TRUNCGAUSS (MODEL ZEROSUBPOP) of $1 \mathrm{G}+2 \mathrm{G}$ and $2 \mathrm{G}+2 \mathrm{G}$ versus $1 \mathrm{G}+1 \mathrm{G}$ mergers of 0.3 (0.26) and $0.05(0.04)$ respectively, with $99 \%$ upper limits of $0.57(0.48)$ and $0.18(0.13)$. When $V_{\text {esc }} \sim 300 \mathrm{~km} \mathrm{~s}^{-1}$, we find that GW190521 is most likely of $2 \mathrm{G}+2 \mathrm{G}$ origin, with 1200:1 and 700:1 odds in favor of being $2 \mathrm{G}+2 \mathrm{G}$ versus $1 \mathrm{G}+1 \mathrm{G}$ using MODEL TRUNCGAUSS and MODEL
ZEROSUBPOP, respectively, with both spin models favoring $2 \mathrm{G}+2 \mathrm{G}$ over $1 \mathrm{G}+2 \mathrm{G}$ origin at $\sim 3.5: 1$. For both models, we find that GW190602, GW190620_030421 (GW190620), GW190706, and GW190519 are most likely of $1 \mathrm{G}+2 \mathrm{G}$ origin, favored over $1 \mathrm{G}+1 \mathrm{G}$ origin at $>10: 1$ odds. Using MODEL ZEROSUBPOP, we also find that GW190517_055101 $(\mathrm{GW} 190517)$ is favored to be of $1 \mathrm{G}+2 \mathrm{G}$ over $1 \mathrm{G}+1 \mathrm{G}$ origin at $>10: 1$ odds.

\section{Conclusions}

GW observations have demonstrated that black holes merge to form more massive black holes (Abbott et al. 2016b). If these merger products form new binaries, they may again merge as a detectable GW source. It is necessary to consider this hierarchical merger channel when using catalogs of GW sources to make inferences about the physics of black hole formation. For example, inference of the location of the lower edge of the pair-instability mass gap, which could potentially constrain nuclear reaction rates (Farmer et al. 2020) or beyond Standard Model physics (Croon et al. 2020; Straight et al. 2020; Baxter et al. 2021), using detections of black holes in the $\gtrsim 50 M_{\odot}$ regime would be contaminated by the presence of $2 \mathrm{G}$ black holes. In order to distinguish between $1 \mathrm{G}$ and $2 \mathrm{G}$ black holes, we must account simultaneously for the shapes of $1 \mathrm{G}$ and $2 \mathrm{G}$ populations and the relative rate of hierarchical mergers. Here, we apply the analysis of Kimball et al. (2020b) to 44 BBHs in GWTC-2, and self-consistently infer a black hole population that accounts for $1 G+1 G, 1 G+2 G$, and $2 \mathrm{G}+2 \mathrm{G}$ binary mergers, as well as the relative branching ratios between them, in order to identify candidate hierarchical mergers in the current catalog of GW sources.

We find the following, assuming our nominal globular cluster model with $M_{\mathrm{c}}=5 \times 10^{5} M_{\odot}$ and $r_{\mathrm{c}}=1 \mathrm{pc}$.

1. The 44 events in GWTC-2 are best modeled when allowing for hierarchical formation channels. For MODEL TRUNCGAUSS and MODEL ZEROSUBPOP, we find Bayes factors of 5 and 7, respectively, in favor of including hierarchical components.

2. At least one BBH in GWTC-2 contains a $2 \mathrm{G}$ black hole with 99\% and $96 \%$ probability using MODEL TRUNCGAUSS and MODEL ZEROSUBPOP, respectively. 
3. The two binaries that are most likely to contain a 2G black hole are GW190519 and GW190521, with 1.2:1 and 2.0:1 odds, respectively, of being $1 \mathrm{G}+2 \mathrm{G}$ versus $1 \mathrm{G}+1 \mathrm{G}$ assuming MODEL TRUNCGAUSS. Using MODEL ZEROSUBPOP, we find that both events have approximately equal odds of being $1 \mathrm{G}+2 \mathrm{G}$ and $1 \mathrm{G}+1 \mathrm{G}$.

4. The relative rates of hierarchical mergers are dependent on how the $1 \mathrm{G}+1 \mathrm{G}$ spin is modeled. Using MODEL TRUNCGAUSS, the median relative merger rates of $1 \mathrm{G}+2 \mathrm{G}$ and $2 \mathrm{G}+2 \mathrm{G}$ to $1 \mathrm{G}+1 \mathrm{G}$ mergers are inferred to be $5.8 \times 10^{-3}$ and $1.7 \times 10^{-5}$, respectively, with $99 \%$ upper limits of 0.12 and $7.6 \times 10^{-3}$. While using MODEL ZEROSUBPOP, the relative rates drop to $5.3 \times 10^{-3}$ and $1.4 \times 10^{-5}$, with $99 \%$ upper limits of 0.04 and $9.8 \times 10^{-4}$, respectively.

5. Using MODEL TRUNCGAUSS (MODEL ZEROSUBPOP), we find that $99 \%$ of $1 \mathrm{G}+1 \mathrm{G}$ black holes are below $40 M_{\odot}$ $\left(40 M_{\odot}\right)$ and that $99 \%$ of all black holes are below $67 M_{\odot}$ $\left(66 M_{\odot}\right)$.

Since we do not believe that all BBHs come from a single type of cluster, we also consider a range of other typical cluster sizes, demonstrating that results depend upon the assumed escape velocity. For a cluster model with $M_{\mathrm{c}}=10^{6} M_{\odot}$ and $r_{\mathrm{c}}=0.1 \mathrm{pc}$, which has the highest Bayes factor:

1. We overwhelmingly favor models including hierarchical formation channels. For MODEL TRUNCGAUSS and MODEL ZEROSUBPOP, we find Bayes factors of 1400:1 and 25000:1, respectively, in favor of including hierarchical components.

2. At least one BBH in GWTC-2 contains a $2 \mathrm{G}$ black hole with probability $>99.99 \%$ for both MODEL TRUNCGAUSS and MODEL ZEROSUBPOP.

3. GW190521 is most likely of $2 \mathrm{G}+2 \mathrm{G}$ origin, with $1200: 1$ and $700: 1$ odds in favor of being $2 \mathrm{G}+2 \mathrm{G}$ versus $1 \mathrm{G}+1 \mathrm{G}$ using MODEL TRUNCGAUSS and MODEL ZEROSUBPOP, with both models favoring $2 \mathrm{G}+2 \mathrm{G}$ over $1 \mathrm{G}+2 \mathrm{G}$ origin at $\sim 3.5: 1$.

4. We find that GW190519, GW190602, GW190620, and GW190706 are most likely of $1 \mathrm{G}+2 \mathrm{G}$ origin for both MODEL TRUNCGAUSS and MODEL ZEROSUBPOP, favored over $1 \mathrm{G}+1 \mathrm{G}$ origin at $>10: 1$ odds, while GW190517 is favored to be of $1 \mathrm{G}+2 \mathrm{G}$ origin above this threshold for MODEL ZEROSUBPOP.

5. Using MODEL TRUNCGAUSS, the median relative merger rates of $1 G+2 G$ and $2 G+2 G$ to $1 G+1 G$ mergers are inferred to be 0.15 and 0.01 , respectively, with $99 \%$ upper limits of 0.29 and 0.04. While using MODEL ZEROSUBPOP, the relative rates drop slightly to 0.14 and $9.2 \times 10^{-3}$, with $99 \%$ upper limits of 0.25 and 0.03 , respectively.

Our analysis indicates that there are plausible hierarchical merger candidates in GWTC-2, meriting further study.

There are a number of possible extensions to this analysis. Most importantly, we have assumed that all merging binaries are formed dynamically in clusters with a specific mass and density. While illustrative, this is unrealistic as (i) the observed $\mathrm{BBH}$ population may come from a mixture of formation channels including isolated field evolution, and (ii) dynamically formed binaries may occur in a wide range cluster types ranging from young open clusters to nuclear star clusters. An excess of events with aligned spin in GWTC-2 suggests that at least some binaries are assembled in the field (Abbott et al. 2021b), and comparisons of observations with model predictions indicate that a mix of formation channels is probable (Bouffanais et al. 2021; Wong et al. 2021;
Zevin et al. 2021). The potential for multiple formation channels could be accounted for by including an additional mixture model for dynamically formed binaries versus those formed in isolation (Kimball et al. 2020b). Previous analyses have suggested using the distribution of spin orientations or eccentricities to measure the fraction of binaries formed dynamically (Breivik et al. 2016; Nishizawa et al. 2016; Stevenson et al. 2017; Talbot \& Thrane 2017; Vitale et al. 2017; Lower et al. 2018; Gondán \& Kocsis 2019; Romero-Shaw et al. 2019; Abbott et al. 2021b; Zevin et al. 2021). Relaxing the assumption that all dynamically formed binaries form in identical environments requires a model for the distribution of globular cluster properties and other dense environments, e.g., AGNs. It is possible that future GW observations will allow us to directly probe the distribution of cluster masses if we obtain sufficient observations to reconstruct the population of host environments. We leave incorporating these extensions to future work.

The authors thank Kyle Kremer, Carl Rodriguez, Mario Spera, and Zoheyr Doctor for their expert advice in constructing this study, and Isobel Romero-Shaw for comments on a draft manuscript. This research has made use of data obtained from the Gravitational Wave Open Science Center (www.gwopenscience.org), a service of LIGO Laboratory, the LIGO Scientific Collaboration and the Virgo Collaboration. LIGO is funded by the US National Science Foundation (NSF). Virgo is funded by the French Centre National de Recherche Scientifique (CNRS), the Italian Istituto Nazionale della Fisica Nucleare (INFN) and the Dutch Nikhef, with contributions by Polish and Hungarian institutes. This work is supported by the NSF Grant PHY-1607709 and through the Australian Research Council (ARC) Centre of Excellence CE170100004. C.K. is supported supported by the National Science Foundation under grant DGE-1450006. C.P.L.B. is supported by the CIERA Board of Visitors Research Professorship. M.Z. is supported by NASA through the NASA Hubble Fellowship grant HST-HF2-51474.001-A awarded by the Space Telescope Science Institute, which is operated by the Association of Universities for Research in Astronomy, Inc., for NASA, under contract NAS5-26555. E.T. is supported through ARC Future Fellowship FT150100281 and CE170100004. T.D. acknowledges support from the María de Maeztu Unit of Excellence MDM-20160692, by Xunta de Galicia under project ED431C 2017/07, by Consellería de Educacíon, Universidade e Formacíon Profesional as Centros de Investigacíon do Sistema universitario de Galicia (ED431G 2019/05), and by FEDER. This research was supported in part through the computational resources from the Grail computing cluster at Northwestern University-funded through NSF PHY-1726951 - and staff contributions provided for the Quest high performance computing facility at Northwestern University, which is jointly supported by the Office of the Provost, the Office for Research, and Northwestern University Information Technology. The authors are grateful for computational resources provided by the LIGO Laboratory and supported by NSF Grants PHY-0757058 and PHY-0823459. This document has been assigned LIGO document number LIGO-P2000466.

\section{Appendix \\ Inferred Population Hyperparameters}

Here, we include the full sets of inferred population hyperparameter posteriors for MODEL TRUNCGAUSS and MODEL ZEROSUBPOP. The $1 \mathrm{G}+1 \mathrm{G}$ primary mass distribution consists of two components. The first is a truncated power law 


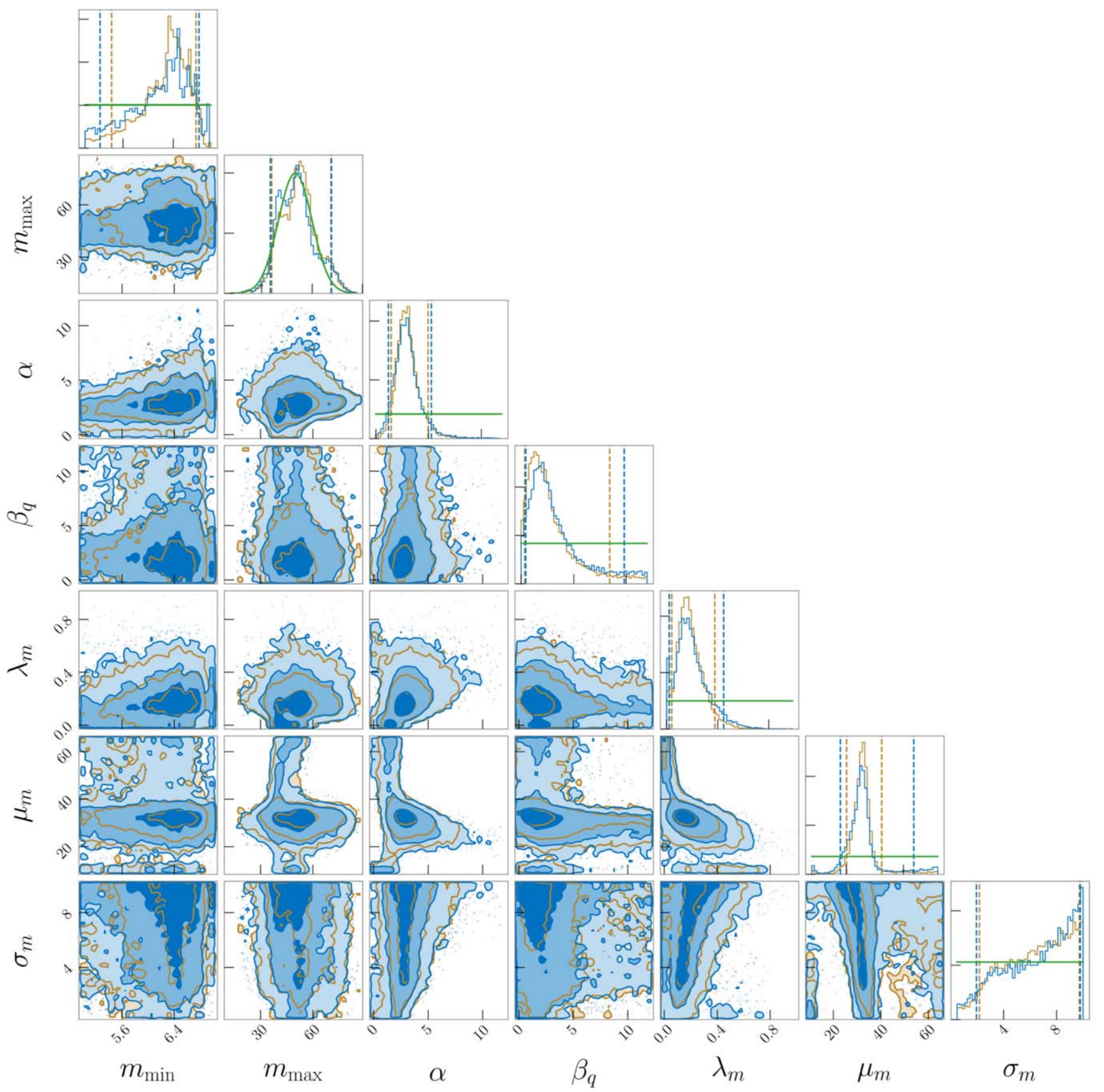

Figure 6. Posterior distributions of the population hyperparameters governing the mass and mass ratio distributions. The dashed lines give the $90 \%$ credible intervals, and the green lines indicate the priors. Results using MODEL TRUNCGAUSS are plotted in orange, and results using MODEL ZEROSUBPOP are plotted in blue.

with minimum mass $m_{\min }$, maximum mass $m_{\max }$, and power-law index $\alpha$. The second is a Gaussian component with mean $\mu_{m}$ and standard deviation $\sigma_{m}$. The mixing parameter $\lambda_{m}$ gives the fraction of BHs drawn from the Gaussian component. The mass ratio distribution is governed by a power law with index $\beta_{q}$. The $1 \mathrm{G}+1 \mathrm{G}$ spin distributions for MODEL TRUNCGAUSS are modeled as truncated Gaussians, with standard deviation $\sigma_{\chi} \in[0.1,10]$ and mean $\mu_{\chi} \in\left[-3 \sigma_{\chi}, 1+3 \sigma_{\chi}\right]$. For MODEL ZEROSUBPOP, we use a Beta distribution with shape parameters $\alpha_{\chi}>1$ and $\beta_{\chi}>1$ and allow a fraction $\lambda_{0}$ of the population to have spins drawn from a delta function at zero:

$$
\begin{aligned}
& \pi\left(\chi \mid \lambda_{0}, \alpha_{\chi}, \beta_{\chi}, 1 \mathrm{G}+1 \mathrm{G}\right)=\lambda_{0} \delta(\chi) \\
& \quad+\left(1-\lambda_{0}\right) \mathrm{B}\left(\chi \mid \alpha_{\chi}, \beta_{\chi}\right) .
\end{aligned}
$$

This zero-spin subpopulation is inspired by simulations of massive stars with efficient angular momentum transfer, where black holes in effective isolation would be born with spins of $\sim 0.01$ (Qin et al. 2018; Fuller \& Ma 2019), and may also describe primordial black holes (De Luca et al. 2020). The $1 \mathrm{G}+2 \mathrm{G}$ and $2 \mathrm{G}+2 \mathrm{G}$ mass and spin distributions are obtained using the transfer functions defined in Kimball et al. (2020b).

In Figure 6, we plot the parameters governing the mass and mass ratio distributions. When using the astrophysically motivated prior on $m_{\max }$ (a Gaussian centered at $50 M_{\odot}$ with standard deviation $10 M_{\odot}$ ), we mostly recover this prior; we do not yet have an informative enough catalog to measure this within our phenomenological model. As in Kimball et al. (2020b), we find that $m_{\max }$ is restricted at small values of the 


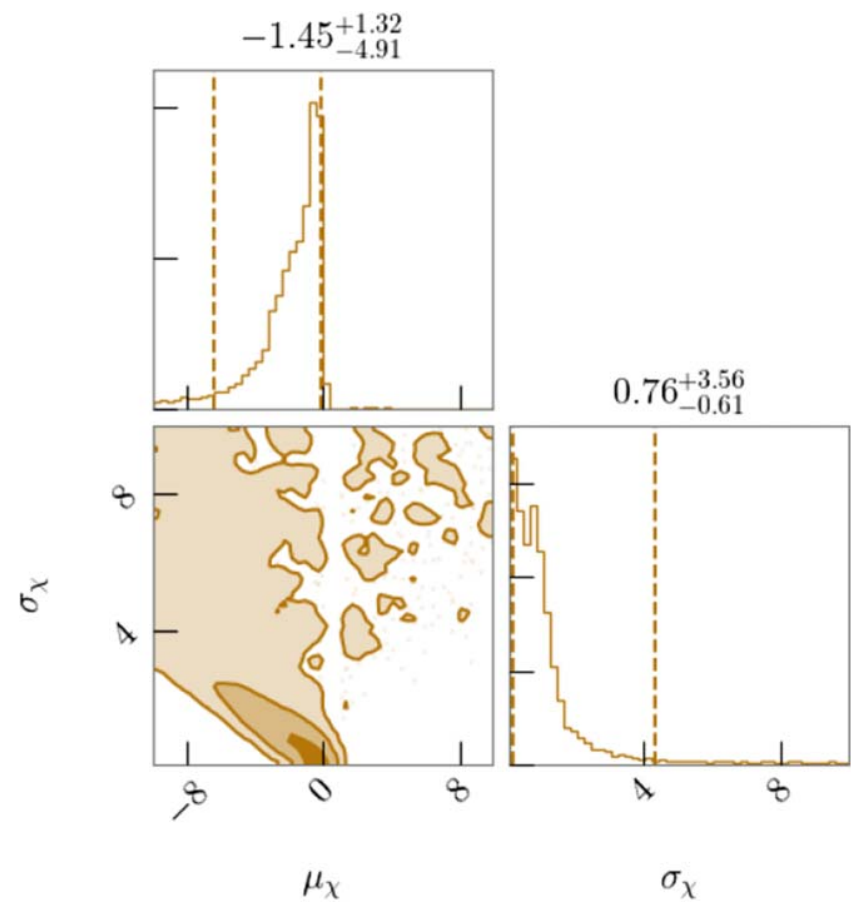

Figure 7. Posterior distributions of the population hyperparameters governing the component spin distributions for MODEL TRUNCGAUSS. The dashed lines give the $90 \%$ credible intervals.

power-law index $\alpha$ where the mass distribution is flatter and more sensitive to the upper mass cut-off. We are able to place stronger constraints on the minimum black hole mass, finding $m_{\text {min }}<6.8 M_{\odot}$ and $m_{\min }<7.0 M_{\odot}$ at the $99 \%$ credible level using MODEL TRUNCGAUSS and MODEL ZEROSUBPOP, respectively. For MODEL TRUNCGAUSS, we find that the Gaussian component of the mass spectrum is well constrained to $\mu_{m}=32.0_{-6.8}^{+8.5} M_{\odot}$. With MODEL ZEROSUBPOP where we infer support for lower relative hierarchical merger rates (Figure 3), we find a long tail at high masses when $m_{\max }$ is low, finding $\mu_{m}=31.5_{-9.0}^{+23.0} M_{\odot}$. With both MODEL TRUNCGAUSS and MODEL ZEROSUBPOP we find a bimodality in the relative hierarchical merger rates, as seen in Figure 3. The peak at higher relative rates is associated with small values of $\sigma_{m}$.
When we restrict the Gaussian component to peak sharply, it is unable to accommodate the highest-mass events as $1 \mathrm{G}+1 \mathrm{G}$ in its tail, and therefore they are fit as hierarchical mergers. If we omit the five highest-mass events in GWTC-2, this peak at higher relative hierarchical rates disappears. Overall, the inferred mass distributions are largely consistent between our two spin models.

In Figures 7 and 8, we plot the parameters governing the component spin distributions for MODEL TRUNCGAUSS and MODEL ZEROSUBPOP, respectively. In MODEL TRUNCGAUSS, we find $\mu_{\chi}<0$, and therefore preference for spin distributions that peak at 0 . In MODEL ZEROSUBPOP, we prefer low values of $\alpha_{\chi}$, which increases support at low component spin, but find that $\beta_{\chi}$ is unconstrained. With MODEL ZEROSUBPOP, we also find that the fraction $\lambda_{0}$ of black holes originating from the zero-spin subpopulation (plotted in Figure 3) is constrained to be less than $0.06(0.12)$ at the $90 \%(99 \%)$ credible level.

We plot the posteriors for the population hyperparameters governing the mass distributions inferred when we assume a flat prior on $m_{\max }$ in Figure 9. Using the flat prior on $m_{\max }$, we no longer constrain the maximum mass cut-off, and the posterior peaks at around $\sim 80 M_{\odot}$. For MODEL TRUNCGAUSS and MODEL ZEROSUBPOP, we constrain the mean of the Gaussian component to be $\mu_{m}=32.1_{-6.1}^{+4.2} M_{\odot}$ and $\mu_{m}=31.6_{-7.3}^{+4.5} M_{\odot}$, respectively. The preference for high $m_{\max }$ means that the high-mass tail on $\mu_{m}$ is no longer required to fit the more massive events in GWTC-2, even though the relative $1 \mathrm{G}+2 \mathrm{G}$ and $2 \mathrm{G}+2 \mathrm{G}$ versus $1 \mathrm{G}+1 \mathrm{G}$ merger rates (as well as the events-wise odds of hierarchical merger) drop by an order of magnitude under the flat prior on $m_{\max }$.

In Figures 10 and 11, we plot the posteriors of the population hyperparameters governing the component spin distributions inferred when we assume a flat prior on $m_{\max }$. We find that the inferred spin distributions are consistent across choices of prior on $m_{\max }$. For MODEL ZEROSUBPOP the fraction $\lambda_{0}$ of BBHs originating from the zero-spin subpopulation is constrained to be less than $0.04(0.09)$ at the $90 \%(99 \%)$ credible level, and is still consistent with $\lambda_{0}=0$. We also find that $\alpha_{\chi}$ and $\beta_{\chi}$ are less constrained; when we allow for high values of $m_{\max }$, it is easier to explain higher mass systems as $1 \mathrm{G}+1 \mathrm{G}$, and hence we do not require a low-spin population to enable high relative hierarchical merger rates. 
The Astrophysical Journal Letters, 915:L35 (14pp), 2021 July 10

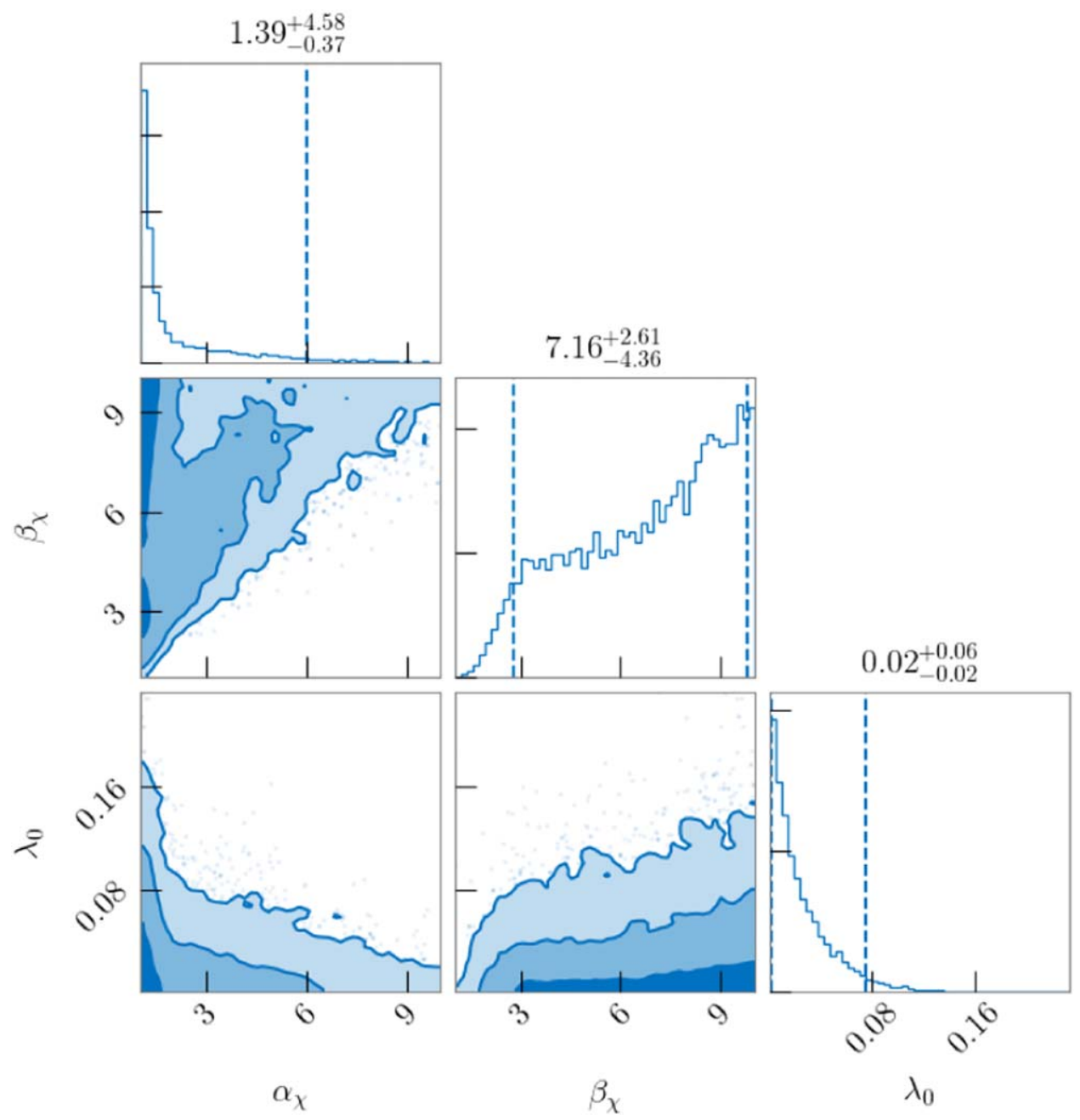

Figure 8. Posterior distributions of the population hyperparameters governing the component spin distributions for MODEL ZEROSUBPOP. The dashed lines give the $90 \%$ credible intervals. 


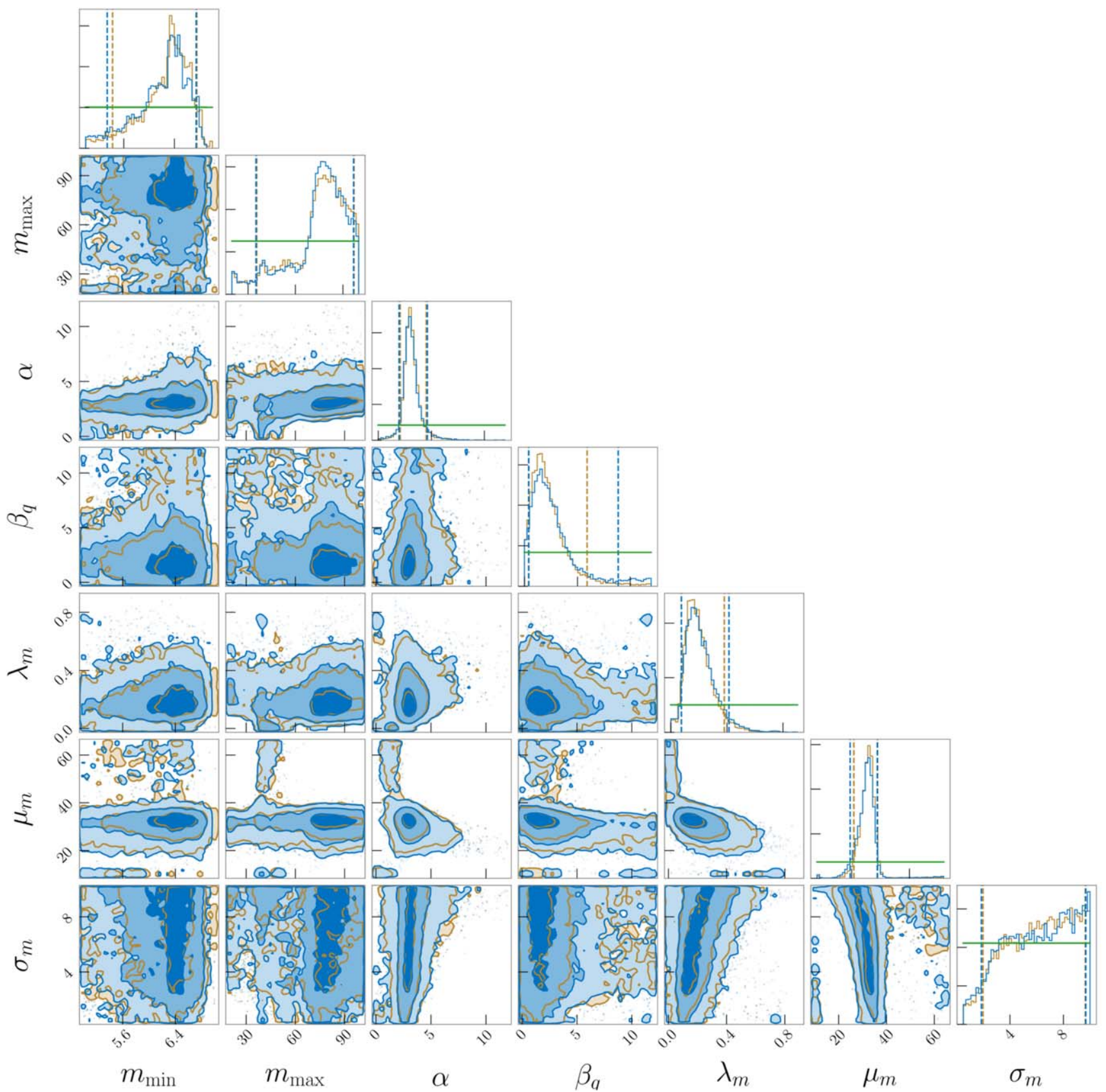

Figure 9. Same as in Figure 6 except with a flat prior on $m_{\max }$. The dashed lines give the $90 \%$ credible intervals, and the green lines indicate the priors. Results using MODEL TRUNCGAUSS are plotted in orange, and results using MODEL ZEROSUBPOP are plotted in blue. 


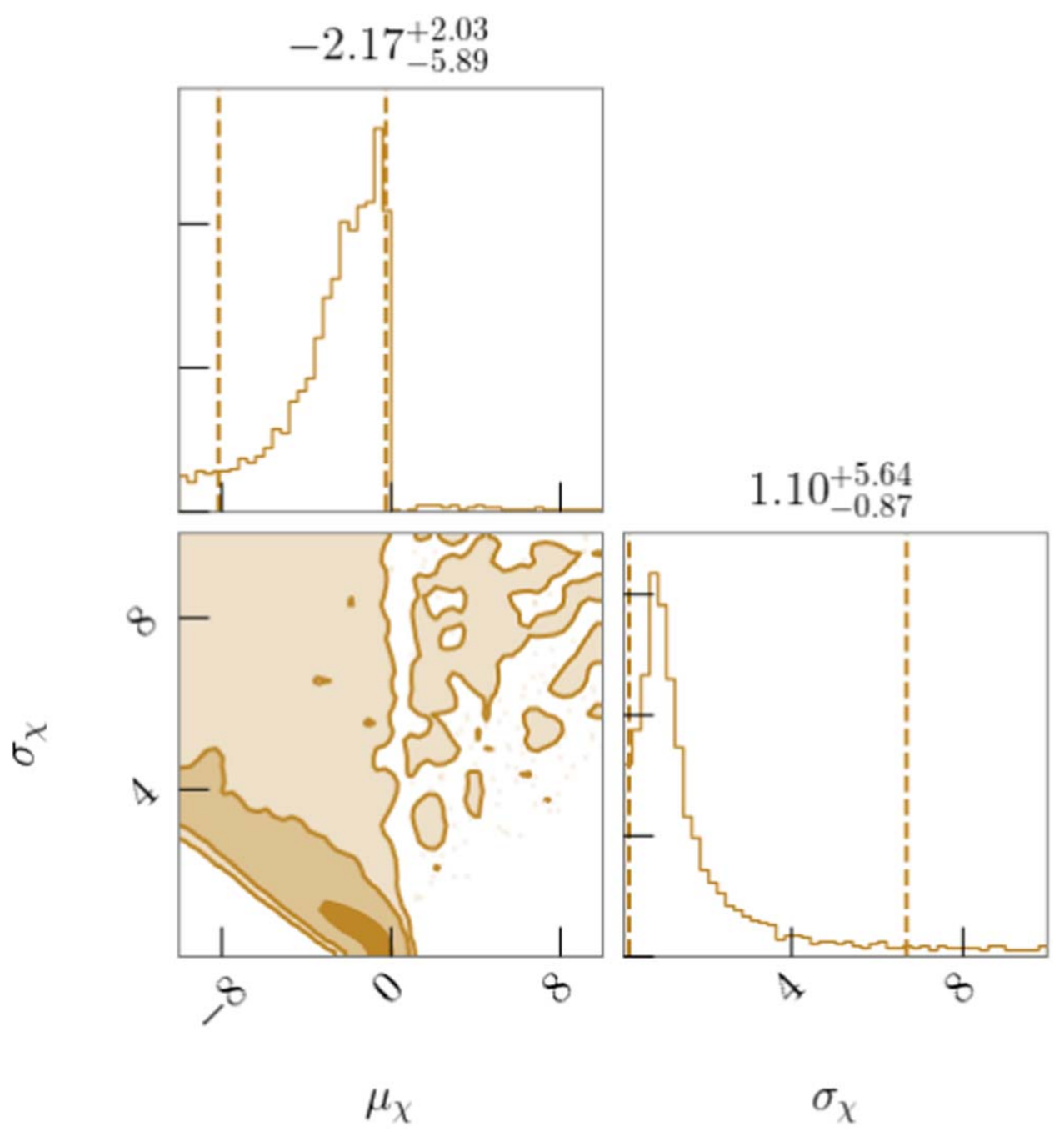

Figure 10. Same as in Figure 7, except with a flat prior on $m_{\max }$. The dashed lines give the $90 \%$ credible intervals. 


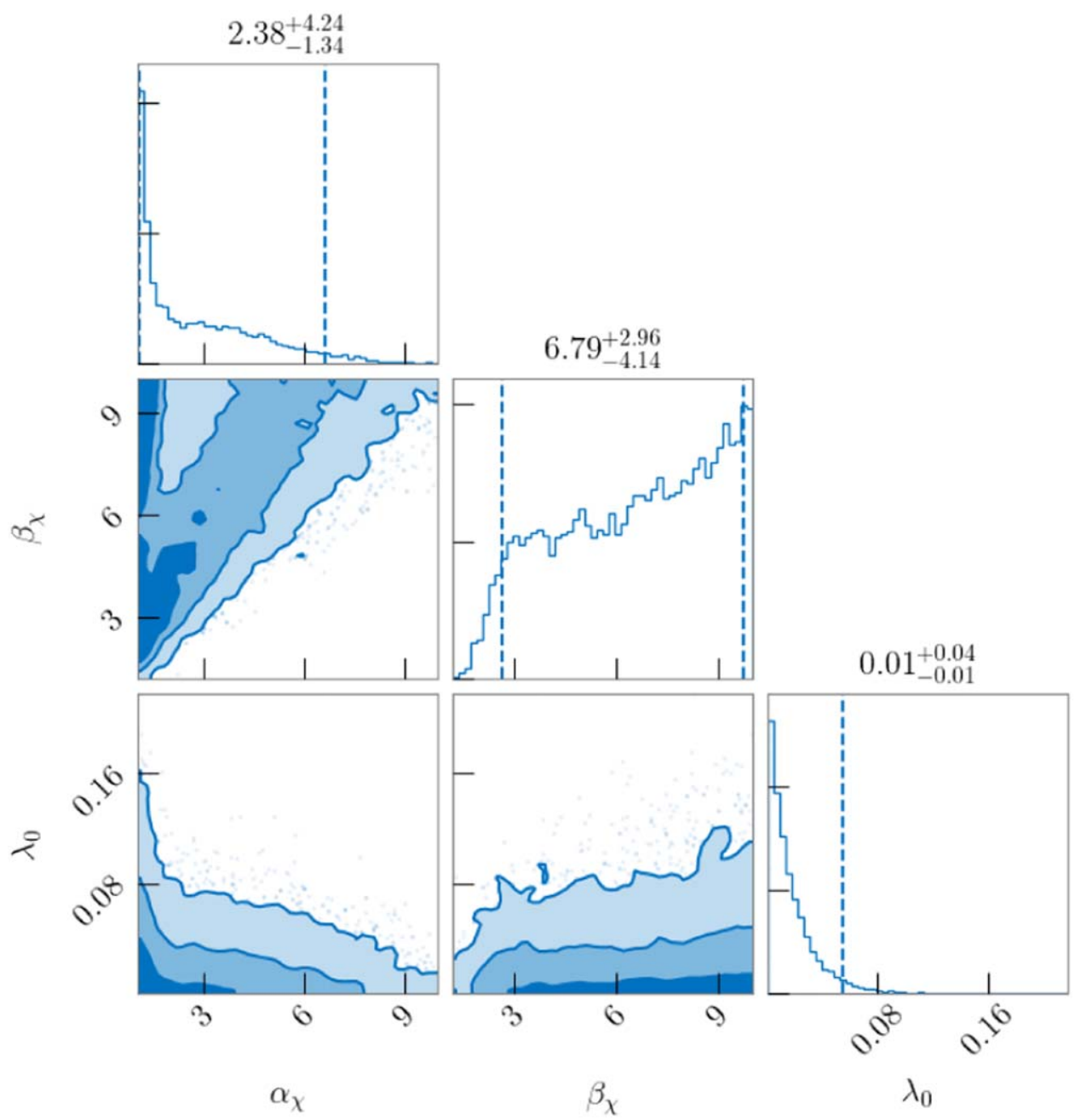

Figure 11. Same as in Figure 8 except with a flat prior on $m_{\max }$. The dashed lines give the $90 \%$ credible intervals.

\section{ORCID iDs}

Chase Kimball $\odot$ https://orcid.org/0000-0001-9879-6884 Colm Talbot (1) https://orcid.org/0000-0003-2053-5582 Christopher P L Berry (i) https://orcid.org/0000-00033870-7215

Michael Zevin (1) https://orcid.org/0000-0002-0147-0835 Eric Thrane (1) https://orcid.org/0000-0002-4418-3895 Vicky Kalogera (1) https://orcid.org/0000-0001-9236-5469 Riccardo Buscicchio (1) https://orcid.org/0000-00027387-6754

Matthew Carney (다 https://orcid.org/0000-0003-4957-6679 Thomas Dent (1) https://orcid.org/0000-0003-1354-7809 Hannah Middleton (10) https://orcid.org/0000-0001-5532-3622 Ethan Payne (1) https://orcid.org/0000-0003-4507-8373 John Veitch (i) https://orcid.org/0000-0002-6508-0713 Daniel Williams (i) https://orcid.org/0000-0003-3772-198X

\section{References}

Abbott, B. P., Abbott, R., Abbott, T. D., et al. 2016a, ApJL, 818, L22 Abbott, B. P., Abbott, R., Abbott, T. D., et al. 2016b, PhRvL, 116, 061102 Abbott, B. P., Abbott, R., Abbott, T. D., et al. 2019, PhRvX, 9, 031040 Abbott, R., Abbott, T. D., Abraham, S., et al. 2020a, ApJL, 896, L44 Abbott, R., Abbott, T. D., Abraham, S., et al. 2020b, PhRvL, 125, 101102 Abbott, R., Abbott, T. D., Abraham, S., et al. 2020c, ApJL, 900, L13 Abbott, R., Abbott, T. D., Abraham, S., et al. 2021a, PhRvX, 11, 021053
Abbott, R., Abbott, T. D., Abraham, S., et al. 2021b, ApJL, 913, L7 Anagnostou, O., Trenti, M., \& Melatos, A. 2020, arXiv:2010.06161 Antonini, F., Gieles, M., \& Gualandris, A. 2019, MNRAS, 486, 5008 Antonini, F., \& Rasio, F. A. 2016, ApJ, 831, 187

Ashton, G., Hübner, M., Lasky, P. D., et al. 2019, ApJS, 241, 27

Banerjee, S. 2021, MNRAS, 500, 3002

Barrett, J. W., Gaebel, S. M., Neijssel, C. J., et al. 2018, MNRAS, 477, 4685 Baumgardt, H., \& Hilker, M. 2018, MNRAS, 478, 1520

Baxter, E. J., Croon, D., Mcdermott, S. D., \& Sakstein, J. 2021, arXiv:2104. 02685

Belczynski, K., Heger, A., Gladysz, W., et al. 2016, A\&A, 594, A97

Bouffanais, Y., Mapelli, M., Santoliquido, F., et al. 2021, arXiv:2102.12495

Breivik, K., Rodriguez, C. L., Larson, S. L., Kalogera, V., \& Rasio, F. A. 2016, ApJL, 830, L18

Bruegmann, B., Gonzalez, J. A., Hannam, M., Husa, S., \& Sperhake, U. 2008, $\mathrm{PhRvD}, 77,124047$

Buonanno, A., Kidder, L. E., \& Lehner, L. 2008, PhRvD, 77, 026004

Campanelli, M., Lousto, C. O., Zlochower, Y., \& Merritt, D. 2007, ApJL, 659, L5

Croon, D., McDermott, S. D., \& Sakstein, J. 2020, PhRvD, 102, 115024 De Luca, V., Franciolini, G., Pani, P., \& Riotto, A. 2020, JCAP, 2006, 044

Di Carlo, U. N., Mapelli, M., Bouffanais, Y., et al. 2020a, MNRAS, 497, 1043 Di Carlo, U. N., Mapelli, M., Giacobbo, N., et al. 2020b, MNRAS, 498, 495

Doctor, Z., Wysocki, D., O'Shaughnessy, R., Holz, D. E., \& Farr, B. 2020, ApJ, 893, 35

Downing, J. M. B., Benacquista, M. J., Giersz, M., \& Spurzem, R. 2011, MNRAS, 416, 133

Farmer, R., Renzo, M., de Mink, S., Fishbach, M., \& Justham, S. 2020, ApJL, 902, L36

Farmer, R., Renzo, M., de Mink, S. E., Marchant, P., \& Justham, S. 2019, ApJ, 887,53 
Farrell, E. J., Groh, J. H., Hirschi, R., et al. 2021, MNRAS, 502, L40 Fishbach, M., Holz, D. E., \& Farr, W. M. 2018, ApJL, 863, L41 Fragione, G., Loeb, A., \& Rasio, F. A. 2020a, ApJL, 895, L15 Fragione, G., Loeb, A., \& Rasio, F. A. 2020b, ApJL, 902, L26 Fragione, G., \& Silk, J. 2020, MNRAS, 498, 4591

Fryer, C. L., Woosley, S. E., \& Heger, A. 2001, ApJ, 550, 372

Fuller, J., \& Ma, L. 2019, ApJL, 881, L1

Gayathri, V., Healy, J., Lange, J., et al. 2020, arXiv:2009.05461

Gerosa, D., \& Berti, E. 2017, PhRvD, 95, 124046

Gerosa, D., \& Berti, E. 2019, PhRvD, 100, 041301

Gondán, L., \& Kocsis, B. 2019, ApJ, 871, 178

González, E., Kremer, K., Chatterjee, S., et al. 2021, ApJL, 908, L29

Gonzalez, J. A., Sperhake, U., Bruegmann, B., Hannam, M., \& Husa, S. 2007, PhRvL, 98, 091101

Hannam, M., Schmidt, P., Bohé, A., et al. 2014, PhRvL, 113, 151101

Heger, A., \& Woosley, S. E. 2002, ApJ, 567, 532

Heggie, D. C., Hut, P., \& McMillan, S. L. W. 1996, ApJ, 467, 359

Holley-Bockelmann, K., Gultekin, K., Shoemaker, D., \& Yunes, N. 2008, ApJ, 686,829

Khan, S., Husa, S., Hannam, M., et al. 2016, PhRvD, 93, 044007

Kimball, C., Berry, C. P. L., \& Kalogera, V. 2020a, RNAAS, 4, 2

Kimball, C., Talbot, C., L. Berry, C. P., et al. 2020b, ApJ, 900, 177

Kinugawa, T., Nakamura, T., \& Nakano, H. 2021, MNRAS, 501, L49

Klencki, J., Moe, M., Gladysz, W., et al. 2018, A\&A, 619, A77

Kremer, K., Spera, M., Becker, D., et al. 2020, ApJ, 903, 45

Kruckow, M. U., Tauris, T. M., Langer, N., et al. 2016, A\&A, 596, A58

Kumamoto, J., Fujii, M. S., \& Tanikawa, A. 2020, MNRAS, 495, 4268

Lousto, C. O., \& Zlochower, Y. 2011, PhRvL, 107, 231102

Lower, M. E., Thrane, E., Lasky, P. D., \& Smith, R. 2018, PhRvD, 98, 083028

Mapelli, M., Dall'Amico, M., Bouffanais, Y., et al. 2021, MNRAS, 505, 339

Mapelli, M., Santoliquido, F., Bouffanais, Y., et al. 2020, arXiv:2007.15022

McKernan, B., Ford, K. E. S., Lyra, W., \& Perets, H. B. 2012, MNRAS, 425,460

Michaely, E., \& Perets, H. G. 2020, MNRAS, 498, 4924

Miller, M. C., \& Hamilton, D. P. 2002, ApJ, 576, 894

Moody, K., \& Sigurdsson, S. 2009, ApJ, 690, 1370

Nishizawa, A., Berti, E., Klein, A., \& Sesana, A. 2016, PhRvD, 94, 064020

Plummer, H. C. 1911, MNRAS, 71, 460

Pretorius, F. 2005, PhRvL, 95, 121101

Qin, Y., Fragos, T., Meynet, G., et al. 2018, A\&A, 616, A28

Rodriguez, C. L., Chatterjee, S., \& Rasio, F. A. 2016, PhRvD, 93, 084029

Rodriguez, C. L., Kremer, K., Grudić, M. Y., et al. 2020, ApJL, 896, L10

Rodriguez, C. L., Zevin, M., Amaro-Seoane, P., et al. 2019, PhRvD, 100, 043027
Romero-Shaw, I. M., Lasky, P. D., \& Thrane, E. 2019, MNRAS, 490, 5210

Romero-Shaw, I. M., Lasky, P. D., Thrane, E., \& Bustillo, J. C. 2020a, ApJL, 903, L5

Romero-Shaw, I. M., Talbot, C., Biscoveanu, S., et al. 2020b, MNRAS, 499, 3295

Roupas, Z., \& Kazanas, D. 2019, A\&A, 632, L8

Sasaki, M., Suyama, T., Tanaka, T., \& Yokoyama, S. 2018, CQGra, 35, 063001

Secunda, A., Bellovary, J., Mac Low, M.-M., et al. 2020, ApJ, 903, 133

Sedda, M. A., Mapelli, M., Spera, M., Benacquista, M., \& Giacobbo, N. 2020, ApJ, 894, 133

Sigurdsson, S., \& Phinney, E. S. 1993, ApJ, 415, 631

Speagle, J. S. 2020, MNRAS, 493, 3132

Spera, M., \& Mapelli, M. 2017, MNRAS, 470, 4739

Spera, M., Mapelli, M., Giacobbo, N., et al. 2018, MNRAS, 485, 889

Stevenson, S., Berry, C. P. L., \& Mandel, I. 2017, MNRAS, 471, 2801

Stevenson, S., Sampson, M., Powell, J., et al. 2019, ApJ, 882, 121

Straight, M. C., Sakstein, J., \& Baxter, E. J. 2020, PhRvD, 102, 124018

Tagawa, H., Haiman, Z., \& Kocsis, B. 2020, ApJ, 898, 25

Talbot, C., Smith, R., Thrane, E., \& Poole, G. B. 2019, PhRvD, 100, 043030

Talbot, C., \& Thrane, E. 2017, PhRvD, 96, 023012

Tang, P. N., Eldridge, J. J., Stanway, E. R., \& Bray, J. C. 2020, MNRAS, 493, L6

Tiwari, V., \& Fairhurst, S. 2021, ApJL, 913, L19

Varma, V., Gerosa, D., Stein, L. C., Hébert, F., \& Zhang, H. 2019, PhRvL, 122,011101

Vigna-Gómez, A., Toonen, S., Ramirez-Ruiz, E., et al. 2021, ApJL, 907, L19

Vink, J. S., Higgins, E. R., Sander, A. A. C., \& Sabhahit, G. N. 2021, MNRAS, 504, 146

Vitale, S., Lynch, R., Sturani, R., \& Graff, P. 2017, CQGra, 34, 03LT01

Webb, J. J., \& Leigh, N. W. C. 2015, MNRAS, 453, 3278

Wong, K. W. K., Breivik, K., Kremer, K., \& Callister, T. 2021, PhRvD, 103 , 083021

Woosley, S. E., \& Heger, A. 2021, ApJL, 912, L31

Yang, Y., Bartos, I., Gayathri, V., et al. 2019, PhRvL, 123, 181101

Zevin, M., Bavera, S. S., Berry, C. P. L., et al. 2021, ApJ, 910, 152

Zevin, M., Berry, C. P. L., Coughlin, S., Chatziioannou, K., \& Vitale, S. 2020a, ApJL, 899, L17

Zevin, M., Pankow, C., Rodriguez, C. L., et al. 2017, ApJ, 846, 82

Zevin, M., Spera, M., Berry, C. P. L., \& Kalogera, V. 2020b, ApJL, 899, L1 